\title{
Deblurring and Denoising of Magnetic Resonance Images using Blind Deconvolution Method
}

\author{
Arunabha Debnath \\ Department of ECE \\ Dronacharya College of \\ Engineering, Gurgaon, India
}

\author{
Hari Mohan Rai \\ Assistant Professor \\ Department of ECE \\ Dronacharya College of \\ Engineering, Gurgaon, India
}

\author{
Chahat Yadav, Anjali \\ Agarwal \& Ankit Bhatia \\ Department of ECE \\ Dronacharya College of \\ Engineering, Gurgaon, India
}

\begin{abstract}
This paper describes the methods to improve the quality of blurred and noisy Magnetic Resonance Images (MRI) due to motion artifact. The main objective of this work is to restore original image from a motion-blurred image due to defocused optical system and motion artifact which is a challenging problem in digital imaging. The blind deconvolution method has been applied to restore the clear image from the blurred and noisy MRI images in this paper. There exist several techniques to restore the original image corrupted by various noise and blurred due to motion artifact, but these methods lack to fetch the originality of image. Blind deconvolution is a method to recover the sharp version of a blurred image when the source of blurring is not known. The aim of this paper is to analyze and evaluate recent blind deconvolution algorithms both theoretically and experimentally for deblurring and noise removal of MRI mges. The MRI data used was corrupted by Gaussian noise and blurred due to various cause (gaussian blurring, out-of-focus blur and motion blur etc). The comparisons of the techniques were done on the basis of Peak signal to noise ratio (PSNR) and Mean structural similarity index (MSSIM). The proposed technique proved to be better over methods used for deblurring and denoising of MRI images.
\end{abstract}

\section{General Terms}

De-Noising, Deblurring, Image Processing.

\section{Keywords}

MRI, Deblurring, Denoising, Blind Deconvolution.

\section{INTRODUCTION}

Images are regarded as a dominant foundation of information and broadly used in abundant fields. Image processing has much application in medical industry, machine vision, and space exploration etc. The image processing field is thus considered to be very complex and of different nature. Due to this, image processing is one of the most challenging areas in mathematics, engineering, medical science, and entertainment industry. Development of computer technology enables us to process images produced by devices such as camera, scanner, ultrasounds, MRI and X-rays to improve their quality, enhance their features, and combine different pieces of information. The clinical MRI data is normally corrupted by noises and blurred during the measurement processes. The aim of image restoration is to obtain clear images from noisy, blurred ones. Image restoration has many applications in field of medical imaging, remote sensing and astronomical imaging etc. The blind image restoration used to reconstruct the original image from corrupted inspection without the knowledge of either the original image or the degradation process. Few more applications, like in some medical imaging problems, the blurring process is unknown and has to be reconstructed together with the image.

If we represent the degradation in a linear model

$$
g(x, y)=f(x, y) \otimes h(x, y)+n(x, y)
$$

where both the original image $f(x, y)$ and the point spreading function (PSF) $\mathrm{h}(\mathrm{x}, \mathrm{y})$ are unknown functions, then the restoration is reduced to a blind deconvolution problem [1]. If the blur kernel is given as a prior, recovering clear image is called a non-blind deconvolution problem; otherwise called a blind deconvolution problem. It is known that the non-blind deconvolution problem is an ill-conditioned problem for its sensitivity to noise. Blind deconvolution is even more ill posed. Because both the blur kernel and the clear image are unknown, the problem becomes under constrained as there are more unknowns than available measurements.

\subsection{Deblurring of Medical Images}

There are two methods to obtain images non-invasively from the interior are PET (Positron Emission Tomography) and SPECT (Single Photon Emission Computed Tomography) [2]. In both cases a radioactive tracer is injected into the patient's body emitting gamma ray photons which are collected by a detector array. The actual image is the output of an inverse reconstruction algorithm. The underlying process is ill conditioned and therefore yields a very noisy and blurred image. Other medical imaging techniques, like MRI or ultrasonic imaging also result in general in very noisy and blurred images. In this paper we found that how the blind deconvolution techniques can be used to improve the quality of the blurred or noisy images. In case of high noise level or a blurred image directed to a miss classification of the pixel.

\subsection{Image Deblurring and Restoration Techniques}

J.Meunier, M. Mignotte, C. Janicki and J.Meunier have compared several restoration techniques on 3D SPECT imaging [3]. They have used two algorithms that are blind deconvolution: Iterative Blind Deconvolution (IDB) and the NASRIF Algorithm. They observed that, the best results can be accomplished using the NASRIF Algorithm. The method involved the information of the support. In their paper the they have used a Marcovian segmentation to estimate the support from a given SPECT image. 
E. Jonsson, Sung-Cheng Huang and T. Chan attempted to integrate total variation regularization into a PET reconstruction algorithm [4]. More complex motion blurring, multi-image based approaches have been proposed to obtain more information of the blur kernel by either actively or passively capturing multiple images on the scene (e.g., Bascle et al. [4], Ben-Ezra and Nayar [5], Lu et al. [6], Raskar [7], Chen et al. [8], Tai et al. [9].

\section{MAGNETIC RESONANCE IMAGING}

An MRI scanner uses the phenomena of nuclear magnetic resonance i.e. it uses magnetic and radio waves, thus there is no risk of exposure to $\mathrm{X}$-rays or any other damaging forms of radiations and a detailed picture of the inside of human body can be obtained. The MRI scanner creates a radio magnetic field which is applied to the body part being exposed. The body's atoms are affected by this field and this field raises their energy level. When the field is removed these atoms relax and they send out radio waves of their own. The MRI scanner picks up these signals and a computer turns them into a picture. Human body consists mainly of water, and water contains hydrogen atoms so the nucleus of the hydrogen atom is often used to create an MRI scan in the manner described above. The tissues having least number of hydrogen atoms (such as bones) turns out dark while the tissues that has many hydrogen atoms (such as fatty tissue) looks much brighter. An MRI scan is able to provide clear pictures of parts of the body that are surrounded by bone tissue, so the technique is useful when examining the brain and spinal cord [10].

\section{LINEAR AND NONLINEAR DEGRADATION MODELS}

The procedure by which the original images are blurred is basically very complex and often unknown. To shorten the calculations the degradation often is modeled as a linear functional. A more detailed discussion of linear and nonlinear degradation models can be found in [10]. For this paper we will assume a linear degradation model:

$$
g(x, y)=\int_{-\infty-\infty}^{-\infty} \int_{-\infty} \mathrm{f}(\xi, v) \mathrm{h}(\mathrm{x}, \mathrm{y}, \xi, v) d \xi d v+\mathrm{n}(\mathrm{x}, \mathrm{y})
$$

where $g(x, y)$ is the blurred noisy image, $f(x, y)$ is the original image, $\mathrm{n}(\mathrm{x}, \mathrm{y})$ is some noise and $\mathrm{h}(\mathrm{x}, \mathrm{y}, \xi, v)$ is the point spread function referred to as psf. In general the point spread function depends on the spacial location i.e. at each point in the image domain there exists a different point spread function. Such a point spread function is called a spatially variant point spread function (SVPSF). Otherwise, the point spread function can be written as $\mathrm{h}(\mathrm{x}, \mathrm{y}, \xi, v)=\mathrm{h}(\mathrm{x}-\xi, \mathrm{y}-\mathrm{v})$, and is called spatially invariant, (SIPSF). In this case the integral term in (2) simplifies and the degradation process is modelled as an ordinary two dimensional convolution,

$$
\begin{aligned}
& g(x, y)=(\mathrm{f} * \mathrm{~h})(\mathrm{x}, \mathrm{y})+\mathrm{n}(\mathrm{x}, \mathrm{y}) \\
& =\int_{-\infty}^{-\infty} \int_{-\infty}^{-\infty} \mathrm{f}(\xi, v) \mathrm{h}(\mathrm{x}-\xi, \mathrm{y}-v) \mathrm{d} \xi \mathrm{d} v+\mathrm{n}(\mathrm{x}, \mathrm{y})
\end{aligned}
$$

the convolution $*$ is, as in the one dimensional case, related to the Fourier transform:

$$
\mathrm{f} * \mathrm{~h}=\mathrm{F}\{\mathrm{f}\} \mathrm{F}\{\mathrm{h}\}
$$

where $F\{f\}$ is used to denote the Fourier transform of function f.

\subsection{Discrete Model}

In the discrete model i.e. both ' $\mathrm{g}$ ' and ' $h$ ' are discrete in equation (2) becomes

$$
g_{i, j}=\sum_{k} \sum_{l} \mathrm{f}_{\mathrm{k}, \mathrm{l}} \mathrm{h}_{\mathrm{i}, \mathrm{j}, \mathrm{k}, \mathrm{l}}+\mathrm{n}_{\mathrm{i}, \mathrm{j}}
$$

Here $f_{i, j}, g_{i, j}$ and $n_{i, j}$ can be regarded as matrix elements of matrices $\mathrm{F}, \mathrm{G}$ and $\mathrm{N}$ respectively and is an element of a four dimensional array. The summations are over all "valid" indices, such that every term that is not zero is taken into account. Usually both the original image and the point spread function have finite support and thus the sum can be expected to be finite. In the spatially invariant case, $h_{i, j, k, l}=h_{i-k, j-l}$, we obtain the discrete equivalent of (3) are;

$$
g_{i, j}=\sum_{k} \sum_{l} \mathrm{f}_{\mathrm{k}, \mathrm{l}} \mathrm{h}_{\mathrm{i}-\mathrm{k}, \mathrm{j}-1}+\mathrm{n}_{\mathrm{i}, \mathrm{j}}
$$

As in the continuous case there is an equivalent formulation using the discrete Fourier trans-form:

$$
f * h=\sum_{k} \sum_{l} \mathrm{f}_{\mathrm{k}, \mathrm{l}} \mathrm{h}_{\mathrm{i}-\mathrm{k}, \mathrm{j}-\mathrm{l}}=d f t\{f\} d f t\{h\}
$$

There are many ways to work with the sum in equation (6). If we suppose that the value of, $g$ and $h$ is 0 outside some supporting region (zero padding) the convolution in (6) will yield a larger image $g$ than $\mathrm{f}$. Zero padding can produce problems when we try to process only a part of the image where we know that the values outside of the processing region is not 0 . On the other hand if we only sum up all valid indices i.e. where both $f$ and $h$ actually have values we will end up with a smaller image g. Note that in this case we do not use zero padding. A third approach is to regard the functions $\mathrm{f}$ and $\mathrm{h}$ as periodic resulting in an infinite and also periodic g. Note that the relation (7) is only valid for periodic $\mathrm{f}$ and $\mathrm{g}$. It is possible to make the periodic case equivalent to the other two cases by using zero padding and using only a subregion of the output of the convolution, at least in a subregion of the picture. This is particularly of interest if the expensive sums are to be replaced by FFTs. The convolution in (6) can also be expressed by a matrix - vector multiplication by rear-ranging the matrices $F, G$ and $N$ to vectors $\mathrm{f}, \mathrm{g}$ and $\mathrm{n}[10]$. (6) becomes:

$$
g=[H] f+n
$$

where the matrix $[\mathrm{H}]$ can be constructed out of the discrete point spread function $\mathrm{h}$ and it has the following Toeplitz structure:

$$
[H]=\left(\begin{array}{l}
h_{0} \\
h \ldots 1 \\
h_{2} \ldots h_{1} \ldots h_{0}
\end{array}\right)
$$

This matrix-vector form can be helpful in the analysis of this problem. Yet, if it is to be used for computations it is most important to make use of the special structure of the matrix, otherwise the computation is very expensive.

\subsubsection{A priori constraints}

As majority of image data signify some kind of a physical object, for example the body of a patient, it can be expected that both the object $f$ and the image $g$ obey a non negativity constraint. Note that this does not necessarily mean that the 
PSF $h$ is positive. As an example think about the approximation of a positive function using the Dirichlet kernel. This kernel is non positive, yet if the value of the function $\mathrm{f}$ is sufficiently far away from 0 then $\mathrm{f} * \mathrm{~h}$ will show the usual under and overshoots, known as Gibbs phenomenon but will stay always positive. Never the less sometimes it makes sense to impose non negativity to the point spread function $\mathrm{h}$

$$
\begin{array}{ll}
f(\xi, v) \geq 0 & \forall(\xi, v) \\
g(x, y) \geq 0 & \forall(x, y)
\end{array}
$$

Equivalently, in the discrete - discrete model:

$$
\begin{array}{ll}
f_{k, l} \geq 0 & \forall k, l \\
g_{i, j} \geq 0 & \forall i, j
\end{array}
$$

In particular, if $\mathrm{g}$ is obtained through an image reconstruction algorithm, the algorithm will usually have been designed to enforce the non-negativity of $g$ through a constraint condition.

\subsection{Point spread functions}

In a linear model the point spread function $\mathrm{h}$ models the blurring of the image. In general this process is not reversible. Theoretically it can be seen from (4) or (7) that the convolutions with the point spread function can be reversed if the spectrum of $\mathrm{h}$ has no zeros in it. Figure 1 shows the original MRI image. Note this is only true for periodic problems. Especially since usually round off-noise and other noise is involved. To illustrate the problem let's look at the convolution as a filtering operation. A filter can be described as a convolution as in (6). In this case the Fourier coefficients are the filter coefficients. If some of the filter coefficients are very small the resulting spectral coefficients of $g$ will be very small and possibly lost to the noise. We have an information loss and the reconstruction of the original function is not possible.

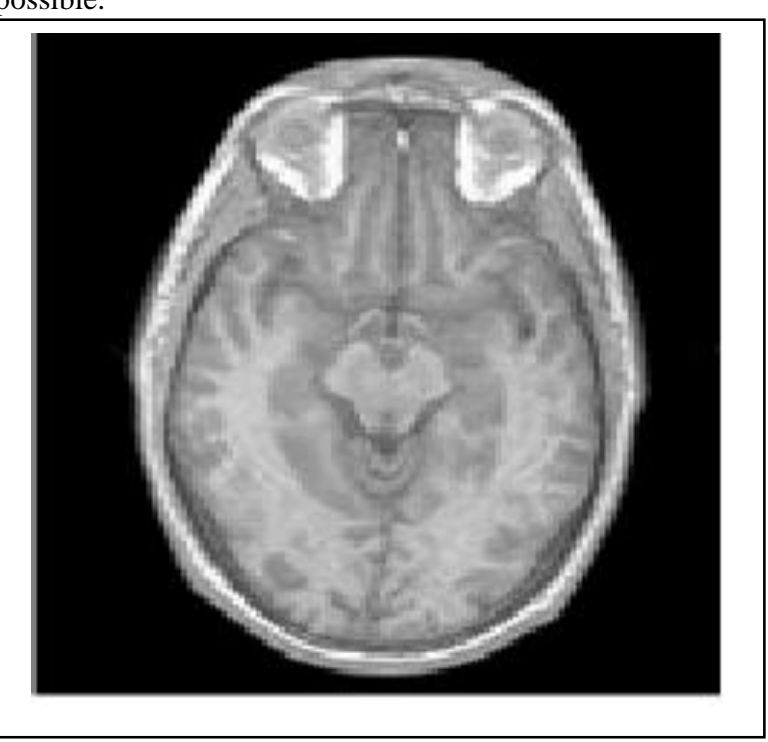

Fig 1: Original MRI Images

\subsubsection{Gaussian Blurring}

The Gauss blur is defined by the following PSF

$$
h(x, y)=\frac{1}{\sigma \sqrt{2 \pi}} \ell \frac{-x 2+y 2}{2 \sigma 2}
$$

usually called variance. This kind of blur occurs for example due to long time atmosphere exposure. Figure 2 shows a Gaussian Blurred MRI image.

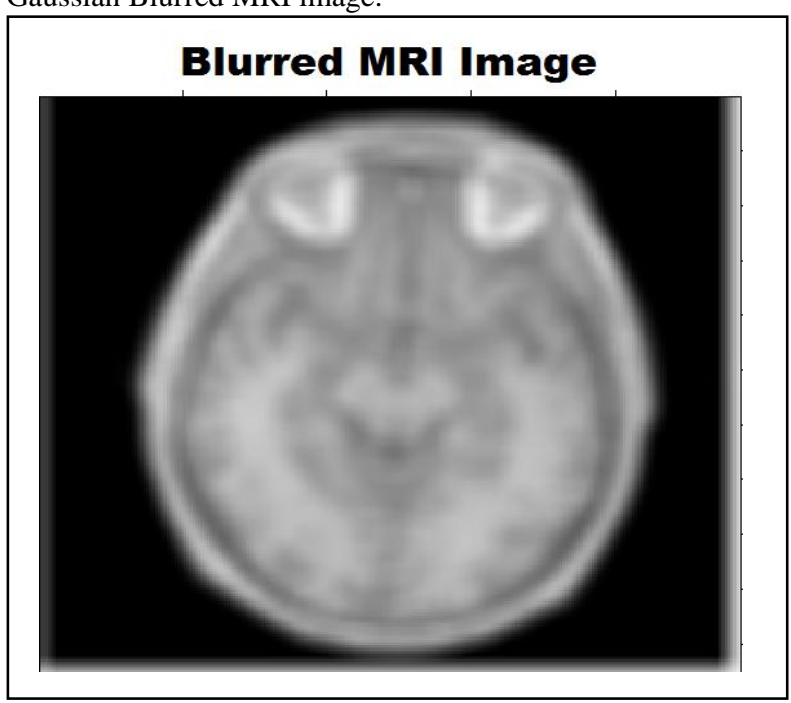

Fig 2: Gaussian blurred MRI image

\subsection{Motion blur}

When an object or the camera is moved during light exposure a motion blurred image is produced. In case of medical application it is very critical to recognize diseases from MRI or X-Ray images. The MRI images might get blurred due to movement of patent or motion artifact. Figure 3 visualizes the MRI image blurred due to motion artifact.

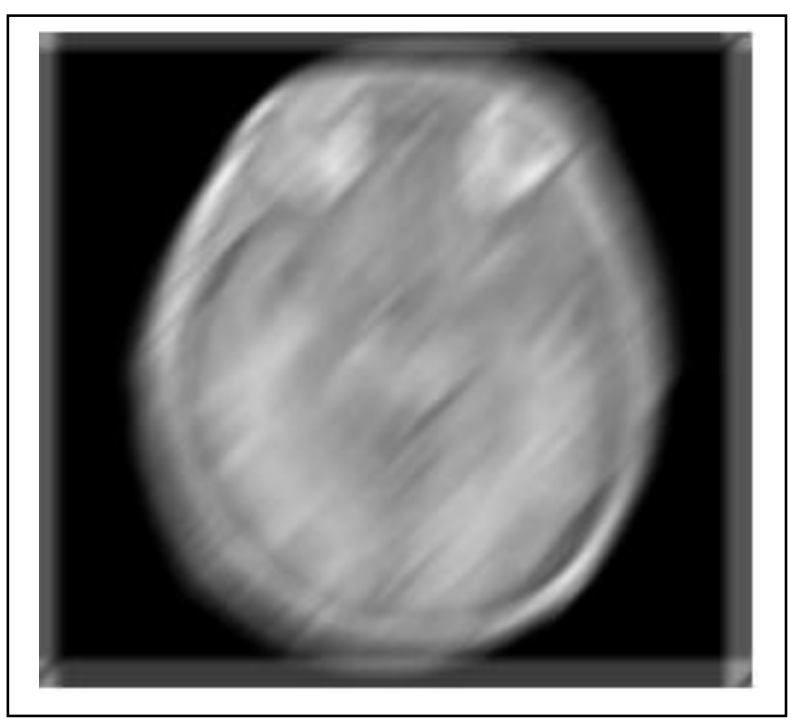

Fig 3: MRI Image with Motion Blur

\subsection{Out-of-focus blur}

This blurring is produced for example by a defocused optical system. It distributes a single point uniformly over a disk surrounding the point. Figure4 illustrates the psf and Figure 4 shows an example of a blurred image due to defocusing of MRI machine. The psf is given by

$h(x, y)=c \begin{cases}1, & \sqrt{\left(x-c_{x}\right) 2+\left(\mathrm{y}-\mathrm{c}_{\mathrm{y}}\right) 2} \leq r \\ 0, & \text { otherwise }\end{cases}$

where $\sigma$ is a parameter of the Gaussian, in statistics it is 
where $\mathrm{r}$ is the radius and $\left(c_{x}, c_{y}\right)$ is the center of the out of focus psf. The scaling factor $\mathrm{c}$ has to be chosen such that $\iint h(x, y) d x d y=1$ i.e. in the continuous case

$$
c=\frac{1}{\pi r 2}
$$

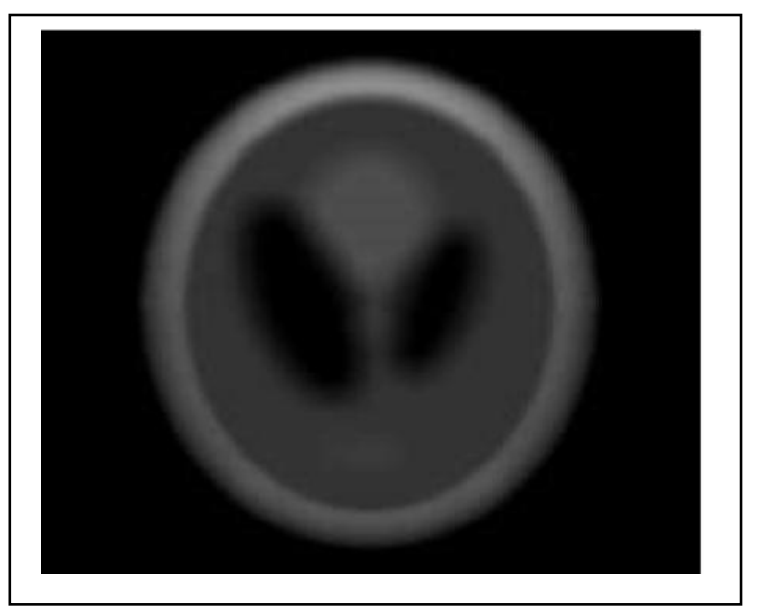

Fig 4: Out of Focus MRI Image

\subsection{Noise}

The primary model for the noisy signal or image is basically of the following form:

$$
I(n)=f(n)+\sigma e(n)
$$

This is the simple noise model where $\mathrm{e}(\mathrm{n})$ is a Gaussian white noise between $\mathrm{N}(0,1)$ and the level of noise is supposed to be equal to 1 .

The objective of denoising of image is to suppress the noise part of image I and to recover the f. From a statistical viewpoint, the model is a regression model over time and the method can be viewed as a nonparametric estimation of the function $\mathrm{f}$ using orthogonal basis. We know that the noise level of a MRI images can be expected to be very high (10\% for MRI). The random distribution of the noise is usually also unknown. Figure 5 shows an example of a MRI image with $10 \%$ Gaussian noise.

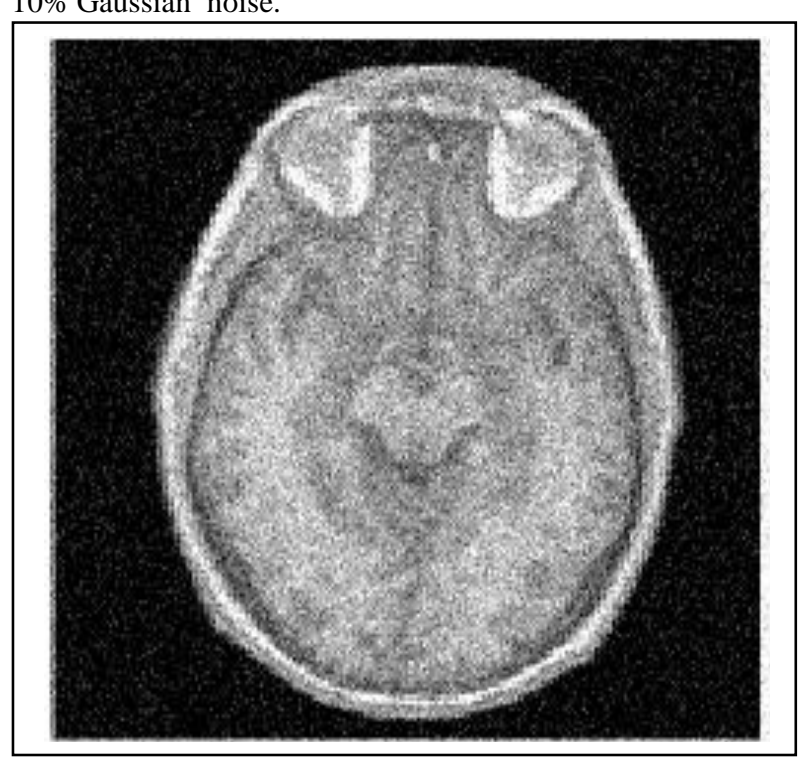

Fig 5: MRI image corrupted by Gaussian noise

\section{EXPERIMENTAL RESULTS}

In this section the proposed technique (Blind Deconvolution) was verified for denoising of and Deblurring the actual MRI Image data. First we have added Gaussian noise where noise variance ranges from 0.02 to 0.09 to the MRI image. Then the noisy image was denoised using Wiener Filter, Median Filter, and Adaptive Filter to provide a baseline comparison. Then the MRI was denoised using proposed technique. To evaluate the performance of the methods PSNR and MSSIM value is used as a quality metric. The graphical result is shown in Figure 6, which shows the comparison Output PSNR vs. Noise variance level. The MRI image with motion blur were taken into account and deblurred using Lucy-Richardson Algorith, Regularized Filter, Wiener Filter and Blind Deconvolution Algorithm. The Deblurring result by various techniques have been shown in Figure 8. Figure 7 shows the Denoising Result of MRi image corrupted by Gaussian Noise. It is observed that noise is suppressed significantly in the output image obtained by proposed method. Table I Shows result for Gaussian noise removal of MRI image. A noisy image which is with PSNR of $18.06 \mathrm{db}$ at noise varience 0.01 the proposed technique improves the PSNR to $27.98 \mathrm{db}$, which is the best result amongst PSNR obtained by other methods. The result implied that the proposed technique i.e Blind Deconvolution Algorithm is best for removal of Gaussian Noise while it also preserves the important information in images. The Experimental Result also states that the Blind Deconvolution technique is best for Deblurring of images due to motion artifact.

Table 1. Denoising result for gaussian noise removal of Mri image

\begin{tabular}{lcccc}
\hline \multicolumn{5}{c}{ Wiener Filter } \\
\hline Noise & PSNR & PSNR & MSSIM & MSSIM \\
Level & $(\mathrm{O})$ & $(\mathrm{D})$ & $(\mathrm{O})$ & $(\mathrm{D})$ \\
$\mathbf{0 . 0 2}$ & 18.06 & 24.64 & 0.3221 & 0.6217 \\
$\mathbf{0 . 0 4}$ & 16.23 & 23.27 & 0.2512 & 0.5487 \\
$\mathbf{0 . 0 6}$ & 14.69 & 20.44 & 0.2076 & 0.4557 \\
$\mathbf{0 . 0 8}$ & 13.95 & 19.21 & 0.1564 & 0.4331 \\
$\mathbf{0 . 0 9}$ & 11.24 & 17.72 & 0.1454 & 0.4013 \\
\multicolumn{5}{c}{ Median Filter } \\
$\mathbf{0 . 0 2}$ & 18.06 & 24.58 & 0.3221 & 0.6465 \\
$\mathbf{0 . 0 4}$ & 16.23 & 22.99 & 0.2512 & 0.5461 \\
$\mathbf{0 . 0 6}$ & 14.69 & 21.77 & 0.2076 & 0.4869 \\
$\mathbf{0 . 0 8}$ & 13.95 & 21.01 & 0.1564 & 0.4355 \\
$\mathbf{0 . 0 9}$ & 11.24 & 18.76 & 0.1454 & 0.4001 \\
\multicolumn{5}{c}{ Adaptive Filter } \\
$\mathbf{0 . 0 2}$ & 18.06 & 25.67 & 0.3221 & 0.7008 \\
$\mathbf{0 . 0 4}$ & 16.23 & 20.45 & 0.2512 & 0.5589 \\
$\mathbf{0 . 0 6}$ & 14.69 & 18.65 & 0.2076 & 0.3505 \\
$\mathbf{0 . 0 8}$ & 13.95 & 16.77 & 0.1564 & 0.2957 \\
$\mathbf{0 . 0 9}$ & 11.24 & 15.22 & 0.1454 & 0.2112 \\
$\mathbf{0}$ & Proposed Method (Blind deconvolution) \\
$\mathbf{0 . 0 2}$ & 18.06 & 27.98 & 0.3221 & 0.8243 \\
$\mathbf{0 . 0 4}$ & 16.23 & 24.25 & 0.2512 & 0.6723 \\
$\mathbf{0 . 0 6}$ & 14.69 & 22.71 & 0.2076 & 0.5911 \\
$\mathbf{0 . 0 8}$ & 13.95 & 21.63 & 0.1564 & 0.4534 \\
$\mathbf{0 . 0 9}$ & 11.24 & 20.84 & 0.1454 & 0.4107 \\
\hline
\end{tabular}




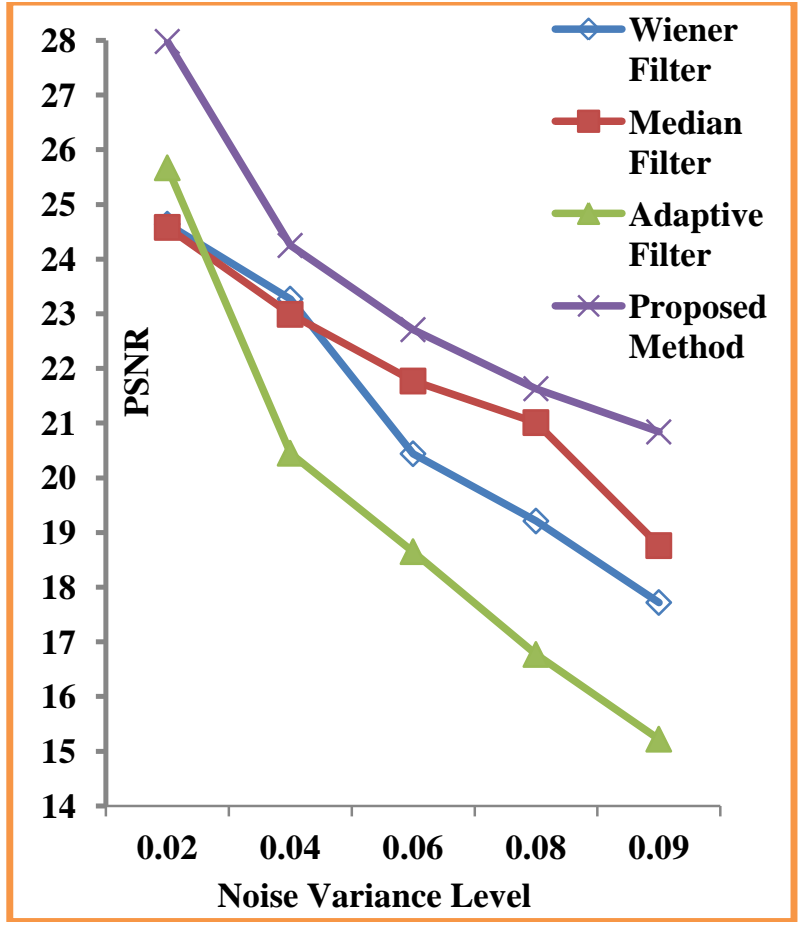

Fig 6: Graphical result for Gaussian noise removal of MRI image

\section{CONCLUSION}

This paper detailed a new image Deblurring and Denoising technique based on Blind Deconvolution Method. Proposed technique was compared with three different techniques (Wiener Filter, Median Filter, and Adaptive Filter) for denoising the MRI image to obtain noise free data. The result obtained by proposed technique was best, among the other existing methods used. The same method was also used for deblurring the MRI image due to motion artifact and result were compared with three other existing methods (LucyRichardson Algorith, Regularized Filter, Wiener Filter). From the simulation result we found that the proposed technique provides the best result for restoring the original data from blur image. The denoising result obtained from proposed method also had a higher PSNR value compared to that from the other methods used for denoising, which (high PSNR) indicates improvement in quality of image. The proposed method was also used to preserve the structure of the image, which is most important concern in image denoising. The technique was thus found to be vigorous for removal of gaussian noise and blur due to motion artifact of images.

\section{REFERENCES}

[1] Zheng Lou, "Blind Deconvolution for Image Restoration Using Recursice Filtering" pp. 2-11, April-2005.

[2] NRC. Mathematics and Physics of Emerging Biomedical Imaging. National Research Council, Institute of Medicine, National Academy Press, Washington DC, 1996. http://www.nas.edu/.

[3] M. Mignotte, J. Meunier, J.P. Soucy, and C. Janicki, "Comparison of deconvolution techniques using a distribution parameter estimation: Application in single positron emission computed tomography imagery", Journal of Electronic Imaging, vol. 11, pp. 5-14, 2002.

[4] B. Bascle, A. Blake, and A. Zisserman. Motion deblurring and super-resolution from an image sequence. In ECCV, pages 573-582, 1996.

[5] M. Ben-Ezra and S. K. Nayar. Motion-based motion deblur- ring. IEEE Trans. PAMI, 26(6):689-698, 2004.

[6] Y. Lu, J. Sun, L. Quan, and H. Shum. Blurred/nonblurred image alignment using an image sequence. In SIGGRAPH, 2007.

[7] R. Raskar, A. Agrawal, and J. Tumblin. Coded exposure photography: Motion deblurring via fluttered shutter. In SIG- GRAPH, volume 25, pages 795-804, 2006.

[8] J. Chen, L. Yuan, C. K. Tang, and L. Quan. Robust dual motion deblurring. In CVPR, 2008.

[9] Y. Tai, H. Du, M. S. Brown, and S. Lin. Image/video deblur- ring using a hybrid camera. In CVPR, 2008.

[10] G.A. Wright, "Magnetic Resonance Imaging", IEEE Signal Processing Magazine, vol.1, pp. 56-66, (1997).

[11] H. C. Andrews and B. R. Hunt. Digital Image Restauration. PRENTICE-HALL SIGNAL PROCESSINH SERIES. PRENTICE-HALL, INC., Englewood Cliffs, New Jersey, 1977. 


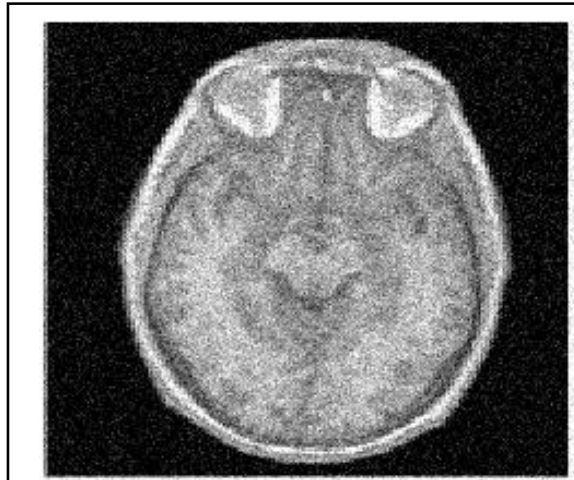

(a) Noisy Image

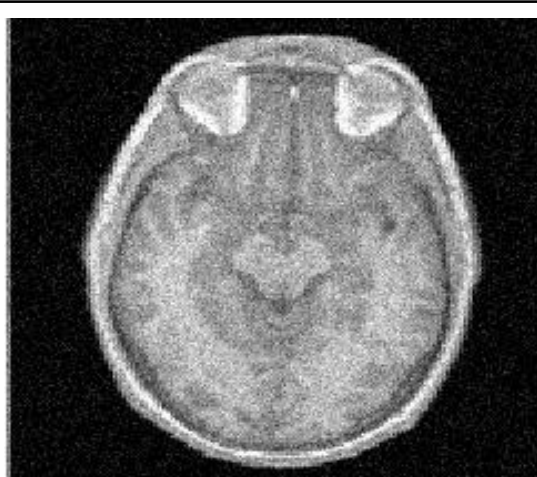

(b) Denoised by Weiner Filter

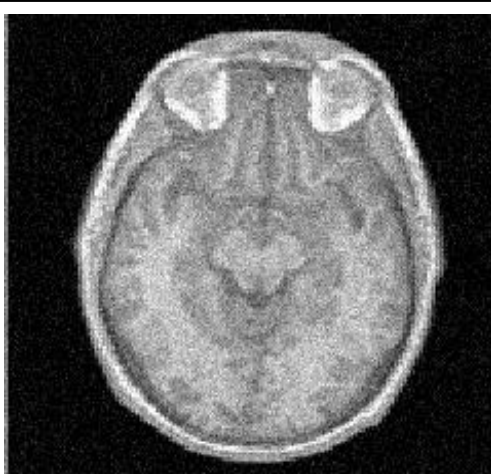

(c) Denoised by Median Filter

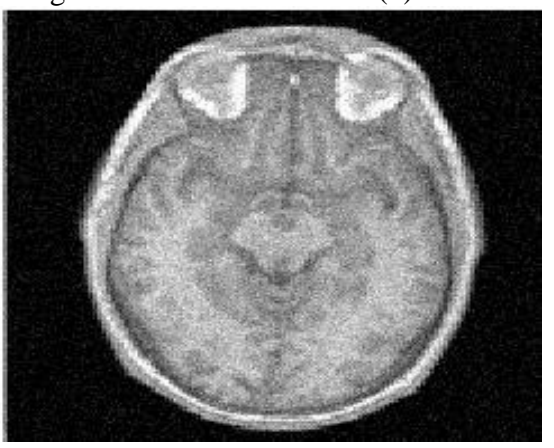

(d) Denoised by Adaptive Filter

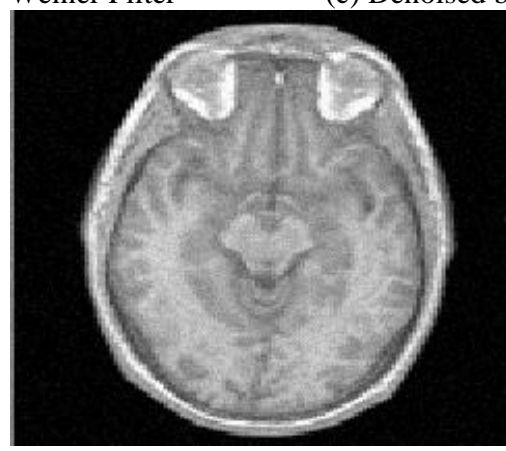

(e) Denoised Proposed method

Fig 7: Gaussian noise removal results of MRI image

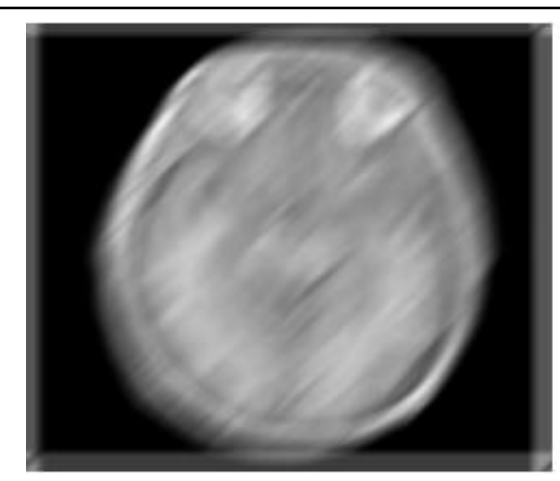

(a) Motion Blurred MRI Image

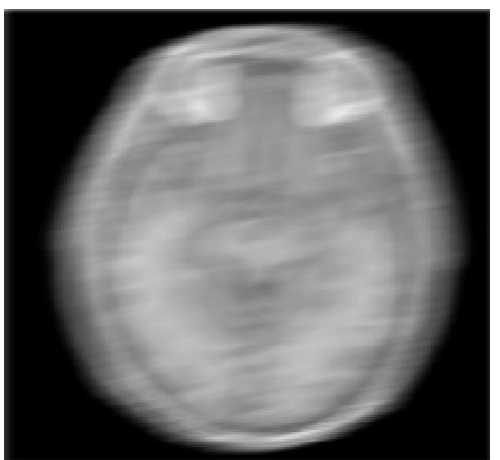

(b) Deblurring using Lucy-Richardson Algorith

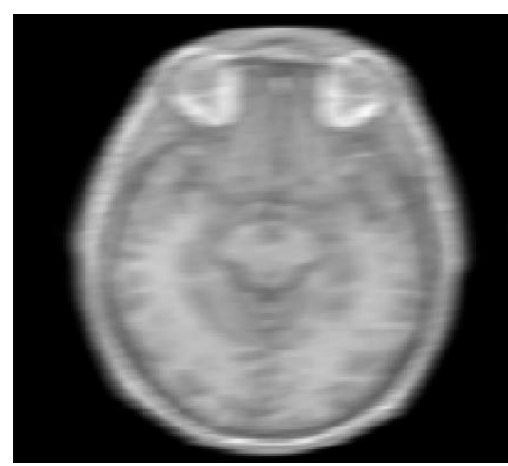

(c) Deblurring using Regularized Filter

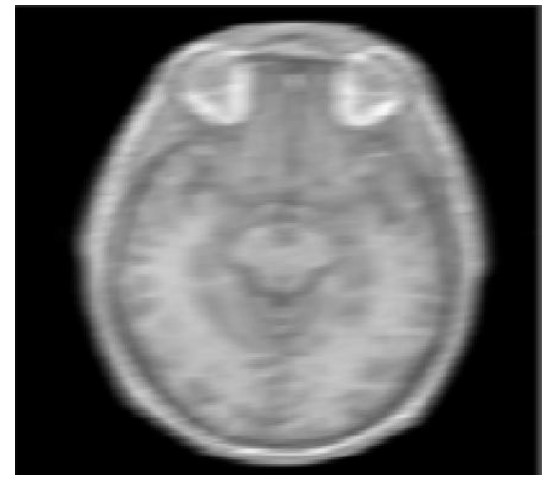

(d) Deblurring using Weiner Filter

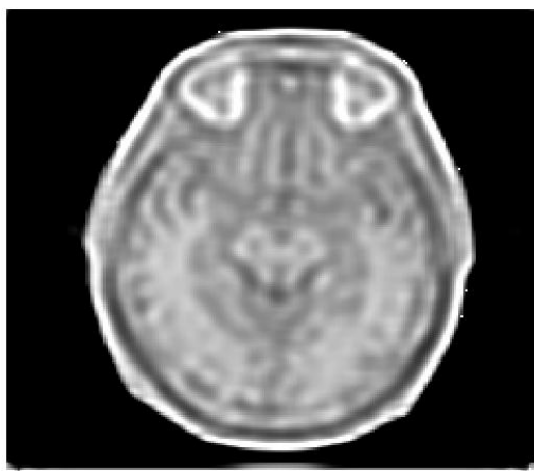

( e) Deblurring by Proposed method (Blind Deconvolution Algorithm

Fig 8: Deblurring results of MRI image due to motion artifact 